\title{
USOS COMPETITIVOS DEL AGUA EN LA CUENCA DEL SEGURA
}

\author{
Alfredo Morales Gil
}

\section{RESUMEN}

El auge de la agricultura hortofrutíco la de exportación y el desarrollo urbano turístico del litoral ha planteado en los últimos treinta años en las provincias de Murcia y Alicante la búsqueda de una solución a la carencia de disponibilidades hídricas, que en una primera etapa se intentó solucionar mediante la captación de aguas subterráneas autóctonas para continuar su aprovechamiento en las tierras del traspaís y finalmente plantear la necesidad de realizar transvases de aguas desde cuencas hidrológicas lejanas, como ha sido el caso de la traída de caudales desde el Tajo al Segura.

Los volúmenes trasvasados durante los siete años de funcionamiento del dicho trasvase han sido de alrededor de $200 \mathrm{Hm}^{3} /$ año, por término medio, aunque las previsiones son de alcanzar los $600 \mathrm{Hm}^{3} /$ año. Con ellos y el ahorro producido en las dotaciones de las huertas tradicionales, que han reducido a $4.000 \mathrm{~m}^{3} /$ hect. y año los consumos, se ha podido alcanzar una superficie transformada de secano en regadío de 113.065 hectáreas, de las cuales unas 30.000 lo han sido utilizando aguas subterráneas y depuradas después de un aprovechamiento urbano e industrial.

\section{RÉSUMÉ}

L'essor de l'horticulture d'exportation et le développement urbain et touristique du littoral depuis une trentaine d'années a impliqué, en ce qui concerne les provinces de Murcie et d'Alicante, la recherche de solutions au manque de disponibilités hydriques qu'on avait, lors d'une premiére étape comblé par le captage d'euaux sonterraines autochtones, pour l'utiliser ensuite sur des terres de l'arriére-pays et finalement affirmer le besoin de réaliser des trasvasements d'eaux de bassins hydroliques éloignés comme en est le cas du Tajo-Segura.

Lors des sept années de fonctionnement de cet aqueduc les volumes trasvasés ont été de $200 \mathrm{Hm}^{3} /$ an en moyenne bien que lon prévoie d'atteindre les $600 \mathrm{Hm} 3 /$ an. De ce fait et gráce á l'économie produite dans les dotations des huertas traditionnelles, qui ont réduit leur consommation á $4.000 \mathrm{~m}^{3}$ / hect., on a pu atteidre une superficie de 113.065 hectares de terrains de culture arides á des terrains irrigables dont 30.000 hect. le sont par les eaux souterraines dépurées aprés une utilisation urbaine et industrielle. 
Las disponibilidades hídricas han ejercido tradicionalmente en España una influencia decisiva en los variados sistemas productivos de cada una de las regiones, esp ecialmente en las tierras del sureste peninsular. Esta situación en los últimos cincuenta años se ha acentuado al aparecer nuevos usos del agua, por lo que las áreas con menos posibilidades han visto agravada la situación y sobre todo han incidido en provocar mutaciones paisajísticas al disputarle a la agricultura su tradicional aprovechamiento hídrico.

Así, se puede afirmar que el agua se ha convertido en un bien escaso a cuidar y a racionalizar su uso hasta en aquellas áreas que secularmente habían sido consideradas como excedentarias desde una apreciación socioeconómica de la cuestión.

El desarrollo de las poblaciones y la aparición de importantes núcleos turísticos e industriales en las provincias de Alicante y Murcia han provocado una lucha sin cuartel para el aprovechamiento de los escasos recursos hídricos autóctonos, evidenciándose inmediatamente que el desarrollo futuro queda hip otecado p or las disponibilidades de agua que se tengan, hasta llegar a cuestionar el modelo de planificación territorial que hoy día se practica.

El auge de la agricultura hortofrutícola de exportación y el desarrollo urbano-turístico del litoral ha planteado, en los últimos treinta años, la búsqueda de una solución a la carencia de potencial de recursos hídricos, que en una primera etap a se intentó solucionar mediante la captación de aguas subterráneas próximas, para continuar la búsqueda en las comarcas del traspaís y, finalmente, plantear la necesidad de realizar trasvases de aguas desde cuencas hidrológicas lejanas, como es el caso de la traída de caudales desde el Tajo al Segura.

Evidentemente, la llegada de aguas del Tajo a la cuenca del Segura en 1979 produjo un despegue decisivo en el desarrollo agrario, urbano-turístico e industrial de las comarcas litorales y prelitorales de las provincias de Murcia y Alicante. La envergadura de esta obra, que se hizo dentro de un marco aparentemente de carácter solidario de la planificación y gestión del agua, en realidad fue la consecución de la actuación de grupos de presión conscientes de las posibilidades de desarrollo socioeconómico de estas comarcas, por otro lado privilegiadas por unas condiciones óptimas climático-térmicas y edáficas.

De esta situación se desprenden dos cuestiones a las que deberá prestarse atención inmediatamente, tal como indica Vera Rebollo (1986), «Por un lado, el tema de las comp etencias en el uso del agua dentro de la misma z ona litoral, donde deberá de hablarse de rentabilidad socioeconómica y de racionalización en el consumo. Pero se plantea además el tema de la importación de caudales, que no está al margen de los susomentados usos. Así la formación de consorcios entre municipios litorales e interiores, para dar solución al problema del abastecimiento de aguas comp orta la prioridad del desarrollo económico costero centrado en el turismo, relegando a un plano secundario las reactivaciones de los municipios interiores, que pasan a ser la periferia suministradora de recursos y mano de obra, en un esquema de articulación del desarrollo regional». 


\section{Demanda de agua para usos agrarios}

El conjunto de tierras murcianas y alicantinas afectadas por la traída de aguas desde la cuenca del Tajo a la del Segura ocupa una superficie de $9.670 \mathrm{~km}^{2}$; de ellas, antes de que se realizase el trasvase, solamente se regaban 159.764 hectáreas, lo que suponía el 16\% del total, de las que 92.670 hectáreas se beneficiaban de las aguas de los ríos Segura $\left(900 \mathrm{Hm}^{3}\right)$, Vinalopó $\left(19 \mathrm{Hm}^{3}\right)$ y $\mathrm{M}$ onnegre $\left(14 \mathrm{Hm}^{3}\right)$, mientras que el resto (67.094) se trataba de regadíos que utilizaban aguas subterráneas. Toda esta zona regada tenía una dotación media de $8.000 \mathrm{~m}^{3} / \mathrm{ha}$ y año.

En los últimos siete años con la llegada de las aguas del «Trasvase Tajo-Segura», disp oniendo p or tanto con una ap ortación media de caudal de $200 \mathrm{Hm}^{3}$ anual, se ha incrementado la superficie regada en 113.065 hectáreas, cifra muy alejada de las previsiones del proyecto inicial que estimaba en una primera fase en $600 \mathrm{Hm}^{3}$ el agua trasvasada y en 269.000 las hectáreas beneficiadas.

La realidad es bastante distinta ya que de estas últimas tierras transformadas en regadío, unas 30.000 hectáreas lo han sido indirectamente mediante la captación de aguas subterráneas y sobre todo mediante la utilización de los vertidos de las depuradoras de aguas residuales de las grandes ciudades, como Murcia, Alicante, Cartagena, Elche... Por otro lado, hay que señalar que esta expansión del regadío en siete años se ha visto impulsada, no sólo por el aumento de may ores caudales para tal fin, sino que también han influido considerablemente la implantación que ha tenido lugar en la última década de métodos de riego localizado p or aspersión, exudación y goteo, que han permitido realizar una economía del agua reduciendo las dotaciones medias a 4.000 o $5.000 \mathrm{~m}^{3} /$ hect. y año. Esto ha permitido, en definitiva, el que la competencia en el uso del agua surgida de las demandas urbano-turísticas e industriales no abocara a estas zonas de regadío a situaciones límites, como sucede en comarcas cercanas del traspaís próximo.

Se ha producido por tanto, un proceso mediante el cual la agricultura tradicional se transforma y se incorpora a unos mecanismos de racionalización en la explotación, con una may or cap italización, mejora tecnológica y dedicación a una serie de cultivos hortícolas cuyos frutos lleguen a los mercados centroeuropeos en los meses invernales, en los que alcanzan sus más altas cotizaciones en base a una débil competencia y unos menores costes de producción, derivadas de las benignas condiciones térmicas que se dan sobre estas tierras en invierno y su acercamiento ráp ido p or carretera a los mercados consumidores.

De esta forma se está articulando un nuevo paisaje rural que contrasta vivamente con el tradicional de «huertas», en el que destacan áreas homogéneas de cultivos de agrios, hortícolas y florales en invernadero, que quedan sup edit ados en su expansión a los recursos hídricos disp onibles. Sin embargo, por falta de una planificación en la comercialización de algunos frutos, como es el caso de los críticos, se han producido crisis de sobreproducción, creando problemas a algunas de las nuevas empresas capitalistas -entidades de crédito- que se habían arriesgado en este tip o de negocios. 


\begin{tabular}{|c|c|c|c|c|c|}
\hline \multirow[t]{2}{*}{ raves } & \multirow[t]{2}{*}{ Dascricte } & \multicolumn{2}{|c|}{ rores $\rightarrow 10 n$} & & \multirow[t]{2}{*}{ 1914ac14 :-4:suse } \\
\hline & & 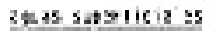 & 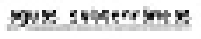 & tesai & \\
\hline Zanos se Zartsment & . $4 i .102$ & & 13.190 & 2.70 & $10.0000 .9 .001 \%$ \\
\hline 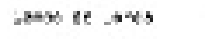 & 2ai,evs & is.tw & $d,(\infty)$ & $8 \rightarrow 000$ & w.tens \\
\hline Earotermals & 70.603 & $\circ, i<0$ & $\sin$ & 4.590 & 3. $\mathrm{x}$ \\
\hline sajs Losdimin & v,tos & & as 201 & 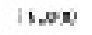 & $14,000,0 ., 000)$ \\
\hline Iros date s-sure & 16.503 & 10.tco & 12.161 & 22.161 & 19.0X triteol" \\
\hline 1ens net, xant & 12:-sej & 1,220 & (. yns & $\therefore, 0$ & $10,0 \times[2,(00) \cdot$ \\
\hline inos tofo secure & $10 . i 60$ & in.tro & a m & 25.on & $16.3 x$ 12.t6ol" \\
\hline bx, the atos & 46.360 & $x .212$ & i.2:s & is.erto & $3 . n x \sim$ \\
\hline $\cos$ ks sticosen & 4.500 & I.stz & 2. 900 & 8.208 & 3.000 \\
\hline '14 & red.tno & \% : 23 & 5i. $\mathrm{Fu}$ & 109.364 & w.oz \\
\hline
\end{tabular}

\section{Demandas urbanas, industriales y turísticas}

Aunque la Ley de Aguas de 1879 ya preveía el uso prioritario del agua para abastecimiento urbano, lo cierto fue que la falta de infraestructura existente en aquella época y en las décadas posteriores en estas tierras murcianas y alicantinas, hasta los años cincuenta y sesenta de esta centuria, evitó el que los escasos caudales disponibles en la cuenca del Segura fuesen utilizados con estos fines, extray endo nada más que unos pequeños volúmenes para el abastecimiento de las grandes poblaciones de Murcia, Alicante, Cartagena, Elche, Lorca y Orihuela; el resto se abastecía de pequeñas fuentes o utilizaban las aguas pluviales que se habían almacenado en aljibes y cisternas.

Esta situación de penuria de dotaciones hídricas a las ciudades se empieza a cambiar en Alicante, cuando en 1907 se constituye la empresa «Canal de Alicante», que obtenía un caudal de 500 libros/seg. de unos pozos situados en el término de Villena. El futuro del puerto de Cartagena y su área industrial estaba impedido por la carencia de agua suficiente para su desarrollo, por lo que en 1946 se creaba la «Mancomunidad de Canales del Taibilla» a fin de trasvasar las aguas del río Taibilla, en la cabecera del Segura, que de una primera aportación de cerca de $6.000 .000 \mathrm{~m}^{3}$ anuales, la mitad eran consumidos por las industrias del Valle de Escombreras e instalaciones portuarias.

Cuando se creó la Mancomunidad de Canales del Taibilla, quedaron englobados en ella la casi totalidad de los municipios murcianos, a los que se le unió posteriormente todos los de las comarcas alicantinas del Bajo Segura, Bajo Vinalopó y Campo de Alicante, con un contingente p oblacional que ascendía en 1981 a unos dos millones de habitantes, con importantes concentraciones urbanas (Murcia, Alicante, Elche, Cartagena, Lorca...), sus respectivas instalaciones industriales y un alargado litoral con importantes concentraciones turísticas, 


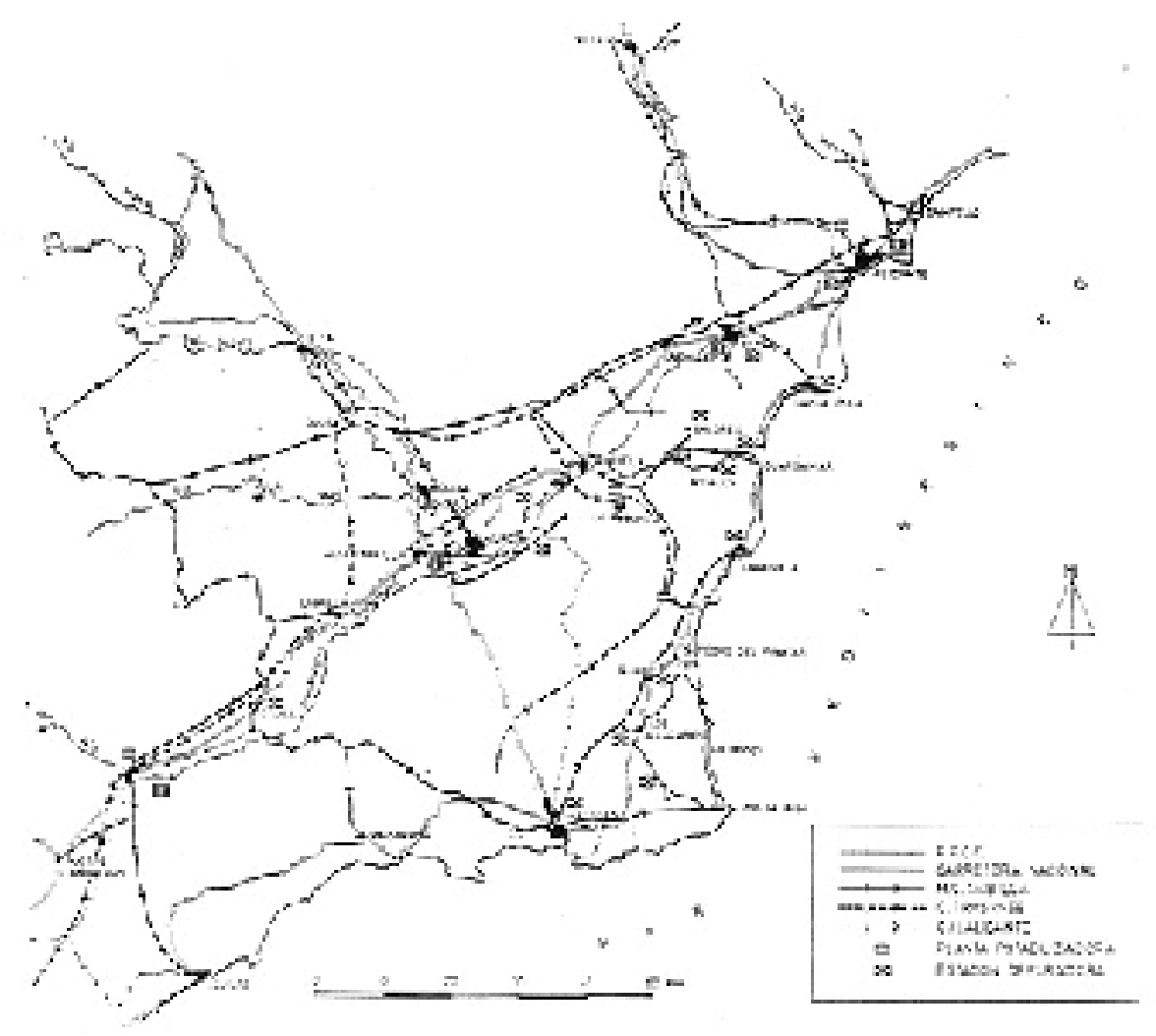

Esquema de las redes de distribución de aguas del trasvase Tajo-Segura y mancomunidad de canales de Taibilla

como Playa de San Juan, Santa Pola, Torrevieja, Campoamor, Ribera del Mar Menor, La Manga, Mazarrón y Aguilas, lo que provocaba que las demandas de aguas se vieran fuertemente incrementadas en los mes es de verano. Por ello, las dotaciones del río Taibilla (56 $\left.\mathrm{Hm}^{3} / a n ̃ o\right)$, las aportaciones de aguas subterráneas de las que disponen algunos núcleos poblaciones y las que se derivaban del Segura se mostraron insuficientes y crearon graves problemas de abastecimiento en la década de los setenta, sobre todo, en época estival, en que la demanda se incrementaba ostensiblemente por parte de las municipalidades con instalaciones turísticas. Así se pasó de un consumo de $34.623 .559 \mathrm{~m}^{3}$ en 1960 a 118.226.790 $\mathrm{m}^{3}$ en 1979, año de la llegada de las aguas del Trasvase Tajo-Segura. En ese mismo período se había pasado de un consumo en las industrias del Valle de Escombreras 
de $2.881 .786 \mathrm{~m}^{3}$ a $6.413 .430 \mathrm{~m}^{3}$. En este último año las restantes industrias cartageneras y las instalaciones portuarias usaron unos $9.500 .000 \mathrm{~m}^{3}$ más.

En 1979 la demanda de Alicante ascendía a 22.000.000 $\mathrm{m}^{3}$ anuales y en Elche era de 12.000.000, con un consumo medio de habitante y día que estaba alrededor de los 200 litros. En la actualidad todo el conjunto de municipios que se abastecen de la «Mancomunidad de Canales de Taibilla» necesita del orden de los $146 \mathrm{Hm}^{3}$ anuales sólo para atender las necesidades de sus habitantes en 1987. A estas demandas hay que unir las de las industrias y sobre todo las turísticas, ya que para un contingente medio de visitantes de unos 750.000 durante sesenta días se puede calcular un consumo adicional de $10 \mathrm{Hm}^{3}$, a los que habría que sumar los consumidos para mantener las áreas de esparcimiento, jardines, campos de golf, piscinas, etcétera, que hoy por hoy es difícil de evalular por no existir ningún estudio estadístico al respecto. En conjunto, se puede estimar que en la actualidad se necesitan del orden de 180 a $200 \mathrm{Hm}^{3} /$ anuales para atender las necesidades urbanas, industriales y turísticas, lo que sup one una cuarta parte de las disponibilidades hídricas que hay en la Cuenca Hidrográfica del Segura.

La llegada de aguas del Trasvase Tajo-Segura, por tanto, ha sido fundamental para permitir asegurar el desarrollo de las industrias y actividades turísticas que se realizan en estas tierras. Si se tiene en cuenta los datos anteriormente expuestos, se puede evaluar en unos $80 \mathrm{Hm}^{3}$ el agua del Trasvase que se destina al consumo urbano, industrial y turístico, lo que representa el $40 \%$ del caudal medio trasvasado, cifra que todavía queda lejos de la prevista de $150 \mathrm{Hm}^{3}$, para cuando el Trasvase se hay a comp letado. Con estos datos se demuestra la imp ortanciadeesta gigant esca obra de ingeniería hidráulica española y su contribución decisiva en el desarrollo turístico de estas tierras. Si bien hay que señalar que esta ordenación y gestión del agua fue la consecuencia de la actuación de grupos de presión que vieron en el agua foránea las posibilidades de asegurar el desarrollo urbano y turístico de estas tierras del sureste peninsular español, que había iniciado su proceso de expansión apoyándose en la explotación de los acuíferos subterráneos, que daba su débil recarga anual estaban condenados a secarse a corto y medio plazo. El agotamiento de caudales en las áreas litorales y la polémica surgida en torno a los trasvases en España, por los desequilibrios territoriales que pueden generar, cuestionan el modelo actual de desarrollo socioeconómico de estas tierras.

\section{Conclusiones}

El modelo desarrollista que se ha implantado en toda la zona afectada por las aguas del trasvase «Tajo-Segura» tiende a programar un crecimiento económico planteado en términos cuantitativos. Por ello habrá que plantearse una programación de planificación cualitativa que conjugara todas las demandas de agua y permitiese que se hiciera un uso más racional de ella, a fin de no tener que estar dependiendo de un aumento continuado de más volúmenes, lo cual es imp osible de asegurar. Habría que empezar por buscar un equilibrio entre recursos naturales disponibles y agricultura, urbanización e industria. 
La definición del agua como un bien escaso obliga a adoptar medidas que garanticen el equilibrio coste-beneficio desde el punto de vista económico. Para conseguir este último objetivo se imponen actuaciones que eviten los usos abusivos tanto en la agricultura como en los demás aprovechamientos, ajustando el precio del agua a su rentabilidad en cada una de sus aplicaciones.

En definitiva, hay que caminar hacia la planificación y gestión integrada del agua en un ciclo hidráulico único que está coordinado por las mancomunidades y consorcios de consumidores, que permitan el que los caudales detraídos de la agricultura reviertan a ésta una vez depuradas y recicladas después de su utilización para usos urbanos, turísticos e industriales.

\section{BIBLIOGRAFÍA}

BASSOLS COMA, M.: «Consideraciones sobre el agua y la ordenación del territorio» I Congreso Nacional de Derecho de Aguas. Murcia, 1982, pp. 341-353.

BOX AMORÓS, M .: «El trasvase Tajo-Segura», Coloquio sobre Demanda yeconomía del agua en España. Alicante, 1986. Tomo IV.

CALVO GARCÍA-TORNEL, F.: «Le bassin du Segura: une expansión problématique de l'irrigation dans le sud-est de l'Espagne», Reune Géographique des Pirénees et du sud-ouest. 55. F.4 Toulouse, 1984, pp. 477-494.

CONSELLERÍA DE OBRAS PÚBLICAS, URBANISMO Y TRANSPORTES: Libro Blanco del Agua en la Comunidad Valenciana. Valencia, 1985. Sin paginar.

GIL OLCINA, A.: " La propiedad del agua en los grandes regadíos deficitarios del sureste peninsular: el ejemplo del Guadalentín». Agricultura y Sociedad, n. ${ }^{\circ}$ 35, 1985, pp. 203-231.

HERALDO DE ARAGÓN: Historia de los regadíos. Edición de J. Domínguez Lasierra. Zaragoza, 1974, $510 \mathrm{pp}$.

LÓPEZ BERM ÚDEZ, F.: «El trasvase Tajo-Segura», Estudios Geográficos, n. ${ }^{\circ} 135,1974$, pp. 321-330.

LÓPEZ GÓMEZ, A.: «Embalses en los siglos XVI y XVII en Levante», Estudios Geográficos, n. ${ }^{\circ}$ 125. Madrid, 1971, pp. 617-656.

MARTÍN-RETORTILLO BAQUER, S.: «La organización institucional del agua», I Congreso Nacional de Derecho de Aguas, Murcia, 1982, pp. 591-600.

MINISTERIO DE OBRAS PÚBLICAS: Plan Nacional de Obras Hidráulicas. Exposición General Ed. de M. Lorenzo Pardo. Madrid, 1933. Tomo I, 301 pp.

MOLINA IBÁÑEZ, M.: «Demanda urbana, turística e industrial del agua», Coloquio sobre «Demanda y economía del agua en España». Alicante, 1986. Tomo II.

MORALES GIL, A. y JUÁREZ SÁNCHEZ-RUBIO, C.: «Cambio en los usos del agua». Estudios Geográficos, n. ${ }^{\circ}$ 165. 1981, pp. 375-395.

MORALES GIL, A.: «Problemas referentes al Agua en España», IX Coloquio de Geografía, A.G.E., Murcia, 1986, pp. 187-196.

- «Trasvases hídricos en España», Coloquio sobre demanda y economía del agua en España.

Alicante, 1986. Tomo IV.

- «El agua: un bien escaso», Revista El Campo, n. ${ }^{\circ}$ 103, 1986, pp. 31-36.

- «Aprovechamiento conjunto Tajo-Segura y otros trasvases», Revista El Campo, n. ${ }^{\circ} 103,1986$, pp. 47-50.

PÉREZ PICAZO, M. T. y GUY LEMEUNIER: «Agua y coyuntura económica. Las 
transformaciones de los regadíos murcianos (1450-1926). Geocrítica n. ${ }^{\circ}$ 58. Barcelona, 1985, pp. 3-87.

PRESIDENCIA DEL GOBIERNO: Estudios de base para la planificación territorial de la Cuenca del Segura. Ed. Subsecretaría de Planificación. Madrid, 1977, 370 pp.

TORRES MARTÍNEZ, M.: El regadío murciano problema nacional. I.O.Y.A.T.S. Murcia, 1959, $68 \mathrm{pp}$.

VERA REBOLLO, J.: «Los recursos hídricos como factor condicionante del desarrollo turístico en el litoral alicantino», Coloquio sobre Demanda y economía del agua en España. Alicante, 1986. Tomo II. 